\title{
Telemonitoring with Electronic Devices in Patients with a Single Ventricle Anatomy
}

\author{
Peter A. Zartner ${ }^{1}$ Nathalie Mini ${ }^{1}$ Diana Momcilovic ${ }^{2}$ Martin B. Schneider ${ }^{1}$ Sven Dittrich ${ }^{3(1)}$
}

${ }^{1}$ Department of Cardiology, German Paediatric Heart Centre, University of Bonn, Bonn, Germany

2 Department of Cardiology and Pulmonology, University of Bonn, Bonn, Germany

3 Department of Paediatric Cardiology, University of ErlangenNuremberg, Erlangen, Germany

Thorac Cardiovasc Surg 2021;69:e53-e60.

\author{
Address for correspondence Peter A. Zartner, FESC, Department of \\ Cardiology, German Paediatric Heart Centre, University of Bonn, \\ Campus Venusberg 1, 53721 Bonn, Germany \\ (e-mail: pzartner@gmail.com).
}

Abstract
Keywords
- Fontan circulation
- single ventricle
- pacemaker
- AlCD
- total cavopulmonary
circulation
- epimyocardial
- implantation

Background A growing number of patients with a single ventricle anatomy, who had a Fontan palliation as a child, are now reaching adulthood. Many need an epimyocardial pacemaker system with an optional telemonitoring (TM) unit, which evaluates the collected data and sends it via Internet to the patient's physician. There are no data on the reliability and clinical relevance of these systems in this patient group.

Methods We analyzed data in 48 consecutive patients (mean age 18 years, standard deviation 9 years) with a Fontan or Fontan-like palliation who received a cardiac implantable electronic device with a TM unit from Biotronik (Home Monitoring) or Medtronic (CareLink) between 2005 and 2020 with regard to the reliability and clinical relevance of the downloaded data.

Results The observation period was from 4 months to 14 years (mean 7 years, standard deviation 3.9 years). A total of 2.9 event messages (EMs)/patient/month and 1.3 intracardiac electrogram recordings/patient/month were received. Two patients died during follow-up. The combination of regularly arriving statistical data and 313 clinically relevant EMs led to the modification of antiarrhythmic or diuretic medication, hospitalization with cardioversion or ablation, and cortisone therapy to avoid exit block in 21 (44\%) patients.

Conclusion TM is an instrument to receive functional and physiologic parameters of our Fontan patients. It provides the ability to respond early for signs of system failure, or arrhythmia, even if the patient is not experiencing any problems. It is a useful tool to manage this difficult patient population without frequent hospital visits.

\section{Introduction}

For children with single ventricle anatomy, palliative treatment consists of surgically connecting the systemic venous return directly to the pulmonary circulation. This circuit relies on passive flow through the lung bed without an additional driving force of a subpulmonary ventricle., ${ }^{1,2}$ Generally, the circuit is fashioned surgically in a stepwise fashion across childhood and the final palliated state is called the Fontan-Kreutzer circulation or total cavopulmonary received

February 14, 2021

accepted after revision

July 2, 2021
DOI https://doi.org/

10.1055/s-0041-1735479.

ISSN 0171-6425.

\footnotetext{
(C) 2021. The Author(s).

This is an open access article published by Thieme under the terms of the Creative Commons Attribution-NonDerivative-NonCommercial-License, permitting copying and reproduction so long as the original work is given appropriate credit. Contents may not be used for commercial purposes, or adapted, remixed, transformed or built upon. (https://creativecommons.org/ licenses/by-nc-nd/4.0/) Georg Thieme Verlag KG, Rüdigerstraße 14, 70469 Stuttgart, Germany
} 


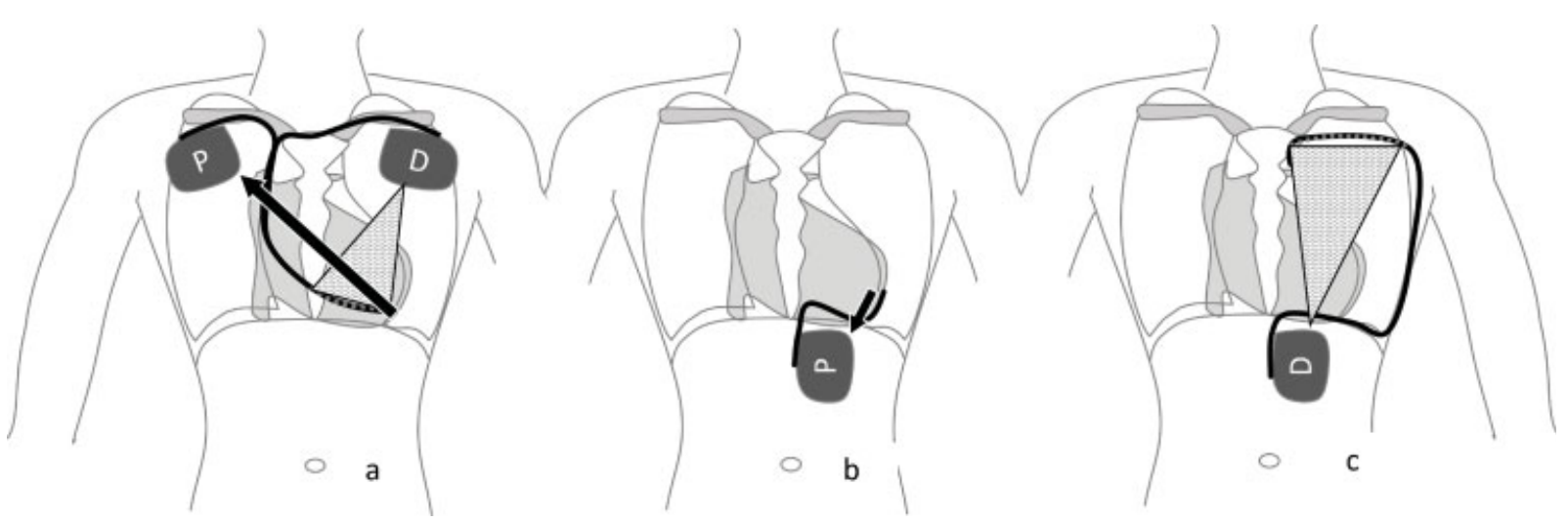

Fig. 1 Different electric vectors and defibrillator fields between transvenous pacemaker (P) and defibrillator (D) systems (a), compared with epimyocardial systems (b, c).

circulation (TCPC). These circuits have evolved since the original descriptions ${ }^{3}$ and have improved symptoms and clinical outcome while reducing complications. ${ }^{4}$ The majority of these patients now reach adult age.

As any form of arrhythmias is badly tolerated in Fontan patients $^{1,5}$ and may rapidly cause cardiac and circulatory failure, the implantation of cardiovascular implantable electronic devices (CIEDs) may be indicated to monitor and direct treatment in these patients. ${ }^{6}$ Since the blood flow of both caval veins drains directly to the pulmonary arteries, this patient group is predisposed to epimyocardial CIED. ${ }^{7}$ This shortens the electric vector between lead tip and CIED and turns it away from the intra- or transthoracic area toward the thoracoabdominal segment (-Fig. 1b). Using single-finger defibrillator leads with epimyocardial automated implantable cardioverter defibrillators (AICDs), the electric field is

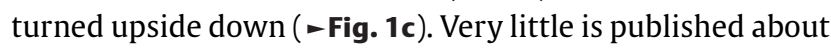
the utility and performance of epimyocardial CIEDs in this patient group. ${ }^{8}$

Modern telemonitoring (TM) systems collect and transmit an increasing number of parameters, reflecting normal function of leads and CIED, underlying heart rate, physical activity, arrhythmic episodes, thoracic impedance (TI) to name a few. These data have the potential to recognize potentially harmful changes within the CIED or the patients' rhythm and circulation, ${ }^{9,10}$ but as yet are unstudied in Fontan patients.

The purpose of this single-center retrospective data analysis was to determine whether the data regularly transmitted by two TM systems can be reliably interpreted, are clinically relevant, and be used for decision-making.

\section{Methods}

\section{Patient Population}

Between 2005 and 2020, a total of 48 patients who had either a TCPC $(n=40)$, a bidirectional anastomosis of the superior vena cava to the pulmonary arteries (Glenn shunt) $(n=2)$, or a "one and half" ventricle repair (Glenn shunt with a pressure reduced pulsed contribution from a hypoplastic ventricle ${ }^{11}$ ) $(n=6)$ received an epimyocardial CIED implant in our clinic (-Table 1).

\section{Indications for a CIED}

Eighteen patients had higher degree atrioventricular block (AVB) as their primary indication for a CIED. Thirteen patients had a sick sinus syndrome (SSS) $(n=10)$ or atrioventricular $(\mathrm{AV})$ dissociation $(n=3)$ with resulting atrial bradycardia. Thirteen patients showed SSS plus AVB and four patients had episodes of atrial tachycardia requiring antiarrhythmic therapy causing clinically relevant bradycardia. One event recorder (ER), 2 atrial (AAI) and 1 ventricular (VVI) pacemakers, 29 double chamber (DDD), and 12 cardiac resynchronization therapy (CRT) systems were implanted. Additionally, three CRT-defibrillators (CRT-D) were used in patients with known episodes of ventricular tachycardia and reduced ejection fraction of less than $40 \%$ (-Table 2 ).

\section{Implantation Procedure}

Bipolar epimyocardial leads, the Greatbatch Myodex 1084-T (Greatbatch Medical, Minneapolis, Minnesota, United States) and the Medtronic 4968 CapSure Epi (Medtronic, Minneapolis, Minnesota, United States) were implanted. For the CRTD, the Medtronic finger lead 6996 Sub-Q Lead (Medtronic)

Table 1 List of patient's main diagnoses

\begin{tabular}{|l|l|}
\hline Diagnoses & $\mathbf{n = 4 8}$ \\
\hline Unbalanced atrioventricular septal defect & 7 \\
\hline Double inlet left ventricle & 8 \\
\hline Double outlet left ventricle & 2 \\
\hline Double outlet right ventricle & 9 \\
\hline Hypoplastic left heart complex & 1 \\
\hline Hypoplastic left heart syndrome & 9 \\
\hline Hypoplastic right heart syndrome & 1 \\
\hline Pulmonary valve atresia & 1 \\
\hline Tricuspid valve atresia & 9 \\
\hline
\end{tabular}


Table 2 List of CIEDs

\begin{tabular}{|l|l|}
\hline CIED & $\mathbf{n}=\mathbf{4 8}$ \\
\hline Biotronik Enitra 8 DR-T & 3 \\
\hline Biotronik Enitra 8 HF-T & 4 \\
\hline Biotronik Entovis DR-T & 9 \\
\hline Biotronik Entovis HF-T & 1 \\
\hline Biotronik Etrinsa 8 DR-T & 7 \\
\hline Biotronik Etrinsa 8 HF-T & 7 \\
\hline Biotronik Iforia 7 HF-T & 1 \\
\hline Biotronik Intica 7 HF-T & 1 \\
\hline Biotronik Itrevia 7 HF-T & 1 \\
\hline Medtronic Adapta ADDR01 & 5 \\
\hline Medtronic Astra XT DR & 2 \\
\hline Medtronic Astra XT SR & 2 \\
\hline Medtronic Sensia SEDR01 & 2 \\
\hline Medtronic Sensia SESR01 & 1 \\
\hline Medtronic Versa VEDR01 & 1 \\
\hline Medtronic Reveal LINQ & 1 \\
\hline
\end{tabular}

Abbreviation: CIED, cardiovascular implantable electronic device.

was used as the shock coil lead and implanted either high in the thorax interpleural or subcutaneous behind the scapula. The CIED was placed subdiaphragmatic behind the fascia musculus rectus (-Fig. $\mathbf{1}$ ).

The apex of the dominant systemic ventricle was the primary location to implant the right ventricular lead. In the case that a second ventricular lead was required, it was placed in the latest contracting region of the ventricle or on the diaphragm side opposite the outflow tract. ${ }^{12}$ The atrial lead was placed close to the sinus node. The two poles of the 4,968 lead were placed $\sim 10 \mathrm{~mm}$ apart from each other. Acceptable lead parameters were pacing threshold $<2 \mathrm{~V}$, impedance between 200 and $1,200 \mathrm{Ohm}$, atrial sensing amplitude $>1 \mathrm{mV}$, and ventricular sensing $>5 \mathrm{mV}$ for bipolar configuration. The equipment implanted was at the discretion of the referring physician.

\section{Data Collection}

All systems were followed online using either the TM "Home Monitoring" (Biotronik, Berlin, Germany) (TM-HM) or the "CareLink" (Medtronic, Inc.) (TM-CL). The TM-HM automatically uploads a dataset every night. The TM-CL requires the patient to activate the monitor to upload data. It was therefore agreed in advance with the patients that the transmission should be performed weekly or more frequently if there were symptoms.

Data collected from daily measurements were considered as statistical data (SD). Additionally, both TM systems were able to evaluate the collected data and generate an event message (EM) according to adjustable limits, so-called exceptions. These limits were programmed according to the agerelated standard values, so that small deviations above daily individual variations would trigger such an EM. In case of an atrial or ventricular tachyarrhythmia, an EM is sent and both systems are able to isolate and send corresponding recordings of the intracardiac electrogram (IEGM).

From the SD set, 16 parameters (which both systems were able to generate and transmit) were evaluated for each of the devices (-Table 3). One group consisted of 10 system-associated parameters including lead impedance, pacing threshold, and sensing amplitude for all connected leads. The other group consisted of six patient-related parameters including mean heart rate, mean atrial heart rate, mean heart rate at rest, patient activity, mean premature ventricular contractions (PVCS) per hour, and the TI, which is supposed to reflect intrathoracic fluid retention, which has the potential to be an important factor in patients with a passive lung perfusion (-Table 3).

\section{Statistics}

Standard statistics were used for mean, standard deviation, and variances. Student's $t$-test was applied to determine if two parameter groups were significantly different with $p \leq$ 0.05 . All calculations, tables, and graphics were calculated in Microsoft Excel 2016.

\section{Results}

In this study, 34 patients received a Biotronik CIED and 14 patents a Medtronic system (- Table 2). A total of 107 pacing leads and 3 shock coil finger leads were implanted ( - Table 4). Follow-up time ranged from 4 months to 14 years (mean 7 years, standard deviation 3.9 years) with a cumulative observation time of 340 patient years. The mean patient age at the time of the latest cardiac surgery was 4 years (standard deviation 3.3 years, minimum 0.3 years, maximum 18 years). The mean age at the time of CIED implantation was 9.6 years (standard deviation 8.6 years, minimum 0.05 years, maximum 29 years). The mean age of all patients at latest follow-up was 18 years (standard deviation 9 years).

Two patients died 2 and 4 years after their latest pacemaker revision with a known history of frequent ventricular extrasystoles and ventricular runs at the age of 21 and 22 years. Both had refused an upgrade to a CRT-D. A postmortem interrogation either locally or online could not be performed because both patients died in another country, so the cause of death remains unknown.

A total of 313 clinically relevant EM, such as atrial tachycardia, atrial flutter or fibrillation, ventricular tachycardia, high rates of PVCs, elevated mean heart rate, and high or increasing $T I$ were received from 18 patients. Combined with information obtained from the daily arriving SD followed online, electrocardiogram (ECG), Holter ECG, and reported clinical symptoms led to therapeutic changes initiated in 21 patients. These consisted of initiating or modifying the antiarrhythmic therapy for atrial flutter $(n=2)$ (-Fig. 2), atrial tachycardia $(n=5)$, and high rate of PVCs $(n=1)$. Messages of high pacing thresholds led to cortisone therapy in three patients. Ten patients showed signs of heart failure with reduced patients' activity, elevated mean heart rates, 
Table 3 Mean parameter values \pm standard deviation for epimyocardial CIEDs in univentricular circulation

\begin{tabular}{|c|c|c|}
\hline & Medtronic epimyocardial CIED $(n=14)$ & Biotronik epimyocardial CIED $(n=34)$ \\
\hline \multicolumn{3}{|l|}{ Systemic parameters } \\
\hline A impedance (Ohm) & $504 \pm 52$ & $524 \pm 200$ \\
\hline A threshold $(\mathrm{V})\left(^{*}\right)$ & $0.9 \pm 0.2$ & $1.0 \pm 0.4$ \\
\hline A sensing $(\mathrm{mV})$ & $3.2 \pm 1.4$ & $4.2 \pm 2.3$ \\
\hline RV impedance (Ohm) & $542 \pm 43$ & $481 \pm 220$ \\
\hline RV threshold $(\mathrm{V})\left(^{*}\right)$ & $1.6 \pm 0.4$ & $1.6 \pm 0.7$ \\
\hline RV sensing $(\mathrm{mV})$ & $12.7 \pm 5.3$ & $10.5 \pm 4.3$ \\
\hline LV impedance (Ohm) & & $426 \pm 218(n=15)$ \\
\hline LV threshold $(\mathrm{V})$ & & $1.5 \pm 0.7(n=15)$ \\
\hline LV sensing $(\mathrm{mV})$ & & $10.9 \pm 4.0(n=15)$ \\
\hline Shock coil impedance (Ohm) & & $57.4 \pm 6.9(n=3)$ \\
\hline \multicolumn{3}{|l|}{ Patient's parameters } \\
\hline Mean PVCs (1/h) & $22.0 \pm 52.0$ & $17.8 \pm 62.0$ \\
\hline Mean atrial HR $(1 / \min )\left(^{*}\right)$ & $80.5 \pm 9.3$ & $80.7 \pm 10.5$ \\
\hline Mean HR (1/min) & $81.1 \pm 8.6$ & $80.1 \pm 9.6$ \\
\hline Mean $\mathrm{HR}$ at rest $(1 / \mathrm{min})$ & $67.7 \pm 10.2$ & $66.1 \pm 8.7$ \\
\hline Patient's activity $(\mathrm{h} / \mathrm{d})$ & $6.5 \pm 1.4$ & $2.8 \pm 1.5$ \\
\hline Thoracic impedance (Ohm) & $54.6 \pm 15.9$ & $50.3 \pm 18.8$ \\
\hline
\end{tabular}

Abbreviations: A, atrial; CIED, cardiovascular implantable electronic device; HR, heart rate; LV, left ventricle; PVC, premature ventricular contraction; RV, right ventricle.

Notes: All parameters were collected online from telemonitoring systems. Empty fields mean that no such device was implemented. Student's $t$-test revealed all parameters were significantly different $(p \leq 0.05)$, except the ones marked with $\left(^{*}\right)$.

Table 4 Mean and standard deviation for threshold and sensing amplitude, calculated for the different epimyocardial pacing leads

\begin{tabular}{|l|l|l|l|}
\hline Lead quality & Atrial lead $(\boldsymbol{n}=\mathbf{4 6})$ & RV lead $(\boldsymbol{n}=\mathbf{4 5})$ & LV lead $(\boldsymbol{n}=16)$ \\
\hline Threshold $(n=107)$ & $1.01 \pm 0.75(\mathrm{~V})$ & $1.54 \pm 0.88(\mathrm{~V})$ & $1.54 \pm 0.55(\mathrm{~V})$ \\
\hline Greatbatch 1084- $(n=43)$ & $1.33 \pm 1.2(\mathrm{~V})$ & $1.63 \pm 1.06(\mathrm{~V})$ & $1.68 \pm 0.67(\mathrm{~V})$ \\
\hline Medtronic 4968 $(n=64)$ & $0.86 \pm 0.38(\mathrm{~V})$ & $1.47 \pm 0.73(\mathrm{~V})$ & $1.35 \pm 0.27(\mathrm{~V})$ \\
\hline Sensing $(n=107)$ & $3.45 \pm 2.32(\mathrm{mV})$ & $10.01 \pm 4.08(\mathrm{mV})$ & $10.17 \pm 4.53(\mathrm{mV})$ \\
\hline Greatbatch 1084-T $(n=43)$ & $2.79 \pm 1.86(\mathrm{mV})$ & $9.09 \pm 2.57(\mathrm{mV})$ & $10.18 \pm 5.26(\mathrm{mV})$ \\
\hline Medtronic 4968 $(n=64)$ & $3.74 \pm 2.46(\mathrm{mV})$ & $10.68 \pm 4.84(\mathrm{mV})$ & $10.17 \pm 3.64(\mathrm{mV})$ \\
\hline
\end{tabular}

Abbreviations: LV, left ventricle; RV, right ventricle.

Note: All 107 leads were bipolar by design.

and increasing TI, which led to adaptation of the individual diuretic medication (-Figs. 3 and 4). These changes were also monitored via TM in the further course. Two Fontan patients who live more than $1,000 \mathrm{~km}$ from the next pacemaker control center were followed up online, directing them to the center in case foreseeable problems occur or reprogramming becomes necessary (lead failure in a CRT-P in one patient).

For the TM-HM system, we received 3.9 (SD 3.3) $\mathrm{EM} /$ patient/month and 1.4 (SD 1.7) recordings/patient/ month, and for the TM-CL system, 1.9 (SD 2.7) EM/patient/ month and 1.1 (SD 1.8) recordings/patient/month.
Shock efficacy of the three epimyocardial CRT-D systems was tested during the implantation procedure, but so far none of the patients suffered from ventricular tachycardia requiring a therapeutic shock. The shock impedance is part of the SD and reported daily by the TM-HM (-Table 3).

Comparing the two groups of cortisone-coated pacing leads used, there is, in regard to the sensing amplitude and the pacing threshold, a significant $(p=0.05)$ but small advantage for the Medtronic 4968 compared with the Greatbatch 1084-T lead (-Table 4 ) independent of the CIED they are connected to. 


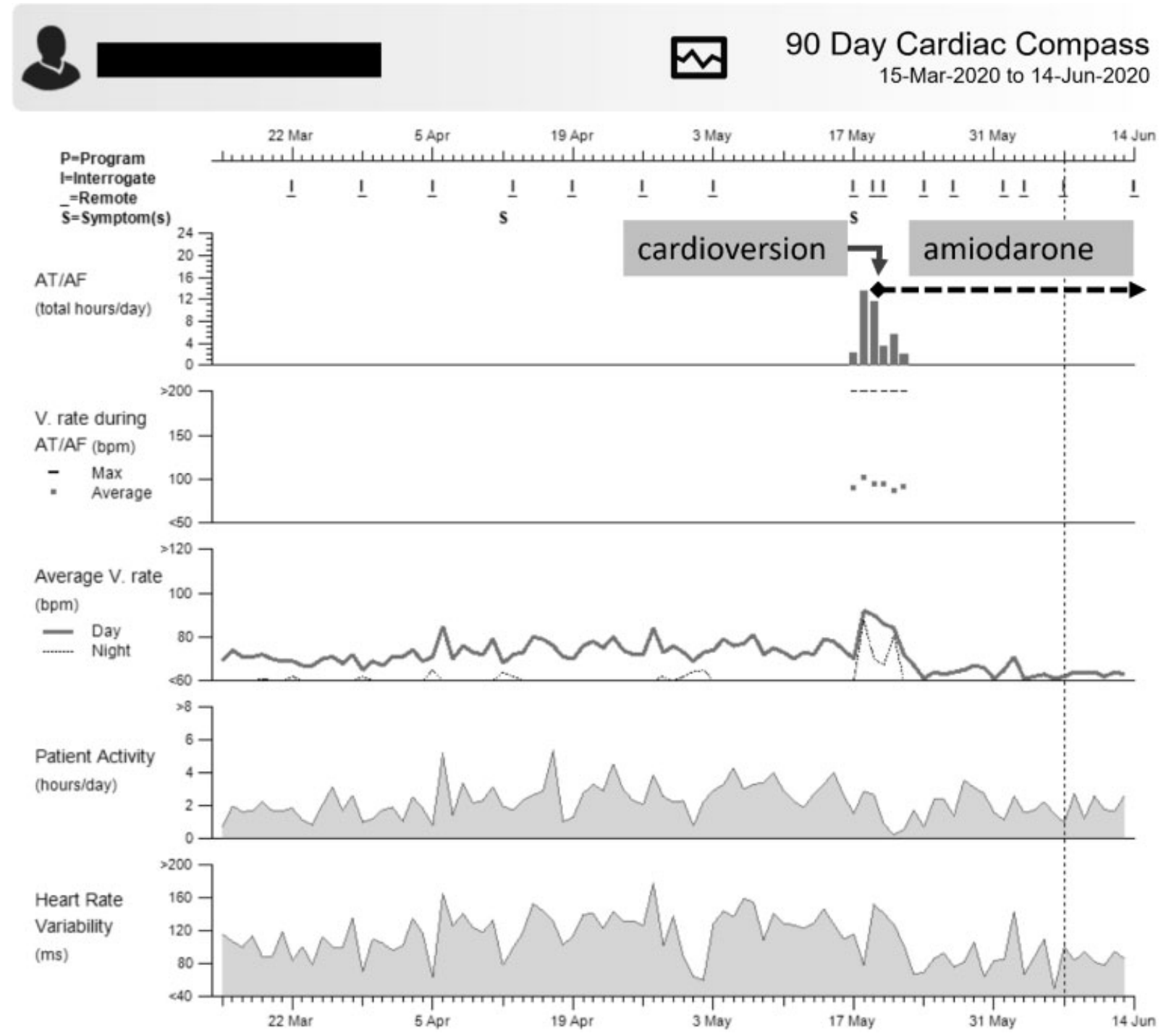

Fig. 2 A 32-year-old man with Fontan circulation on the basis of a tricuspid valve atresia and unclear syncope received a subcutaneous event recorder. Antiarrhythmic therapy was initiated with bisoprolol and amiodaron. Being on holiday, he suffered from dyspnea and dizziness. Telemonitoring revealed atrial flutter. Coming back from holiday 3 days later cardioversion was successful and the amiodaron dose was increased. During the first follow-up days still some self-limiting flutter episodes occurred, but ceased later on.

\section{Discussion}

Longitudinal studies of Fontan patients show that this group has a high complication rate with the need for early and frequent interventions. With age, Fontan circulations deteriorate, and rhythm disorders become more common, occurring in more than one-third of the patients. Over time, Fontan patients will develop irreversible heart failure ultimately needing cardiac transplantation. ${ }^{13}$ Between 23 and $55 \%$ of the Fontan patients receive a pacemaker and 2 and $10 \%$ experience ventricular tachycardia or sudden death with the need of an AICD in 2\%. ${ }^{114}$ Realizing these systems with epimyocardial electrodes results in a different orientation of the electric vectors and shorter range of the electric field at least for unipolar pacing ( - Fig. 1). With the underlying single ventricle dominated morphology and reduced myocardial mass, the electrical vector of the heart may additionally vary in size and orientation. From experience, most of the epimyocardial CIEDs are working perfectly in this setting, if the lead positions are carefully prepared and calibrations at implantation were performed with adequate values close to the ones for transvenous leads.

Low resistance in the pulmonary vascular bed is the most important factor for every Fontan circulation, as this is considered to be the bottleneck. Any elevation in pulmonary vascular resistance, for example, through interstitial pulmonary edema, will increase the central venous pressure and reduce the preload of the systemic ventricle. ${ }^{15}$ This affects compensatory physiological parameters of the heart that can be recorded by modern CIEDs. One of the most important parameters in this regard is the TI. The higher the fluid content of the lungs, for example, due to congestion, the better their electrical conductivity and the lower the 


\section{Thoracic impedance}

Figure shows mean values per week

- Thoracic impedance [ohm]

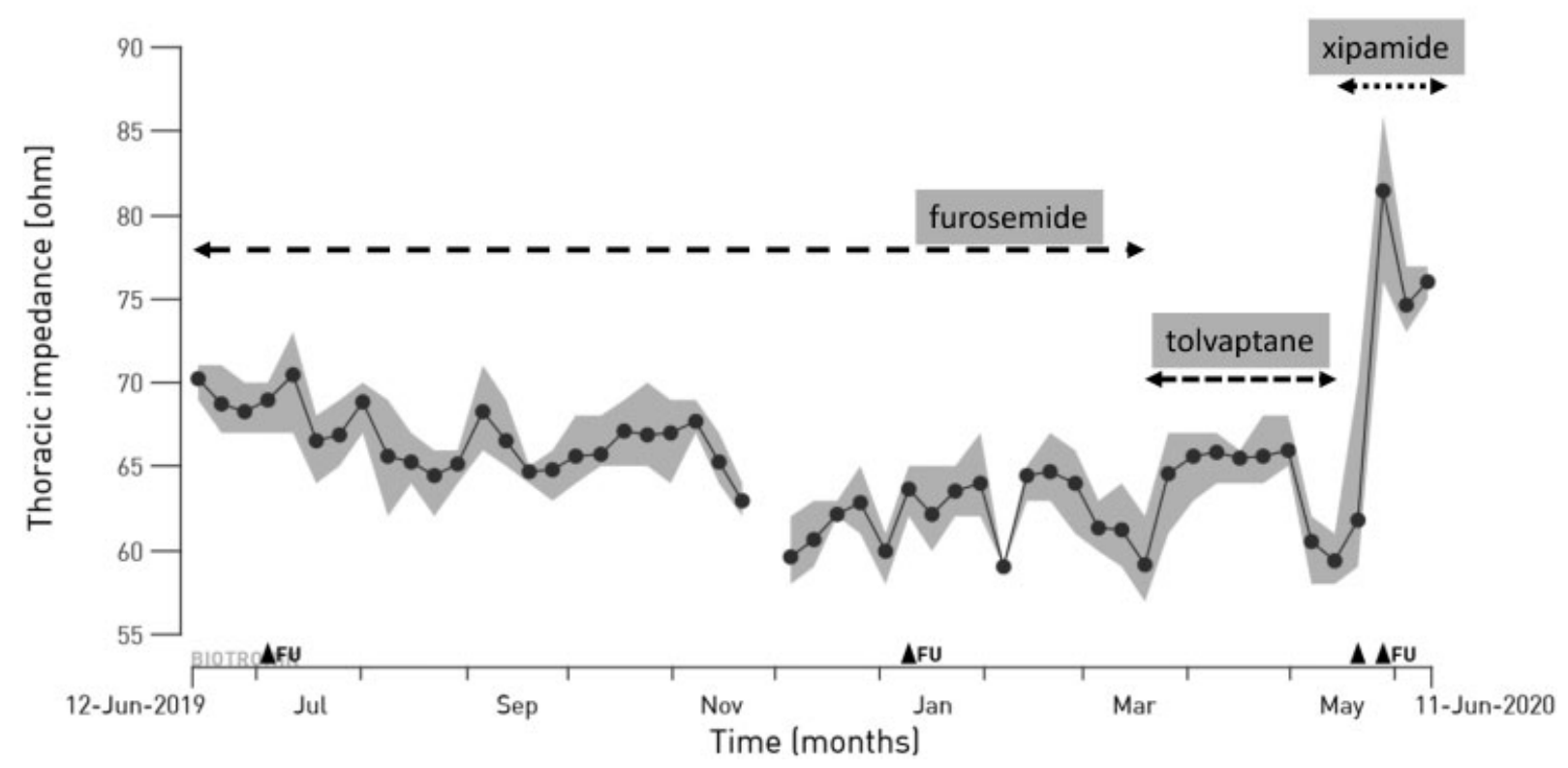

Fig. 3 Thoracic impedance of a patient with an unbalanced atrioventricular channel with a dominant left ventricle and Fontan circulation. The patient is 22 years of age and suffering from persistent ascites, low albumin, and recurrent edema of the lower limbs. Diuretic medication with torasemide proved insufficient and was temporarily supplemented with an additional dose of tolvaptan, which lost its effect after weaning. As an alternative, xipamide showed very effective and was maintained in low dose.

electrical resistance, which can be measured between two poles around the lung tissue.

TI is a parameter measured unipolar between lead tip and CIED cask. In epimyocardial CIEDs, there is only a small part of the lungs between the measurement points, but the absolute values are within the same range known from transvenous systems (- Table 3 ). TI can be altered by diuretic therapy, which in turn makes it appear suitable for therapy control, to anticipate increasing fluid uptake and to manage diuretic medication. The other parameters linked with possible heart failure, as an increase in mean heart rates and reduced patient's activity were used to confirm and undermine the relevance of TI changes.

Episodes of atrial tachycardia, atrial flutter or fibrillation, ${ }^{16}$ were found in more than $20 \%$ of Fontan patients with a modern lateral tunnel and in 30\% with atriopulmonary connection. ${ }^{17}$ Any of these arrhythmias, if unnoticed and untreated, may lead to ascites, pleural effusions, atrial thrombosis, significant AV valve regurgitation, advanced ventricular dysfunction, and heart failure. ${ }^{1}$ Even if these episodes are short and self-limiting, their recurrence rate signals if the antiarrhythmic treatment is effective or if ablation must be considered. All parameters of the epimyocardial systems are in the same numerical range as for transvenous systems, the programmable limits in the TM to start an EM work in Fontan patients identical to other CIEDs. These tachycardic episodes are communicated in all patients under investigation and allow early and controlled treatment.

With the necessary epimyocardial CIED in Fontan patients, an approach with multiple leads in selected locations around the heart is possible and can be easily performed during one of the thoracotomies for Glenn or Fontan repair $^{18}$ even in our younger patients of 3 to 4 years. Even if the leads are not immediately used, any later CIED implantation will be a relatively small intervention if the leads are already in place. With CRT systems and two ventricular leads at the apex and opposite the outflow tract, these systems remain fully functional if one ventricular lead fails without the need for surgery. ${ }^{19}$

Daily (TM-HM) or weekly TM (TM-CL) transmissions of system and patient-related parameters markedly improve the reaction time compared with noncommunicating CIEDs, which are controlled every 3 or 6 months. It must be emphasized that the received TM data must be interpreted as an indication, warning or development in a certain direction, but absolutely require clinical verification. This is especially relevant in a sensitive patient group as Fontan patients, where small changes can lead to decompensation.

\section{Study Limitations}

The diversity of the underlying cardiac malformations may play a nonnegligible role in the measurements described, 


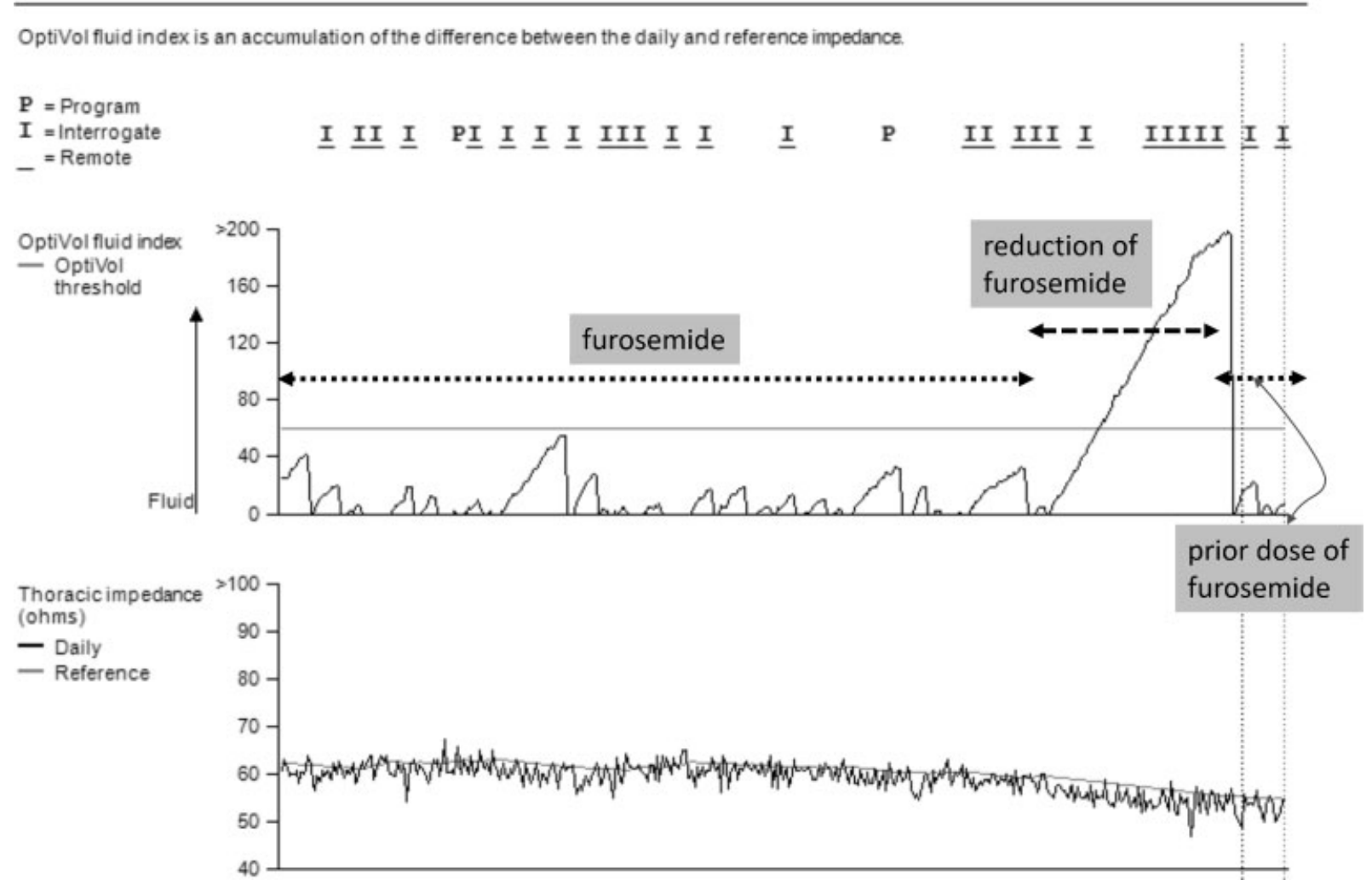

Fig. 4 While reducing furosemide, a 20-year-old woman with a double outlet left ventricle after Fontan palliation showed an OptiVol fluid index above its threshold and clinically an increasing dyspnea (the OptiVol fluid index is an accumulation of the difference between the daily and reference impedance [standard is $60 \mathrm{Ohm}$ ]). As a consequence, furosemide was reinstalled in its prior dosage and the index as well as the respiratory rate improved. This was monitored online with just one in-clinic visit.

but the study group is too small to characterize single diagnoses. The evaluation concentrated on the automated measurements of the CIEDs and the transmitted data. Independent clinical data or symptoms were not included. The observation's time frame lasts up to 15 years and covers at least two technological generations of CIEDs, so not all parameters were monitored over the whole period. The lead material remained unchanged throughout the observation.

\section{Conclusion}

Patients with a single ventricle anatomy and a Fontan or comparable circulation are a high-risk group for circulatory failure even under ideal preconditions. Despite the hardware arrangement is completely different to transvenous systems, modern CIED systems collect an increasing number of parameters, which can trigger further diagnostics in the clinic and to follow the therapeutic measures in this patient group. It provides the ability to respond early if there are signs of system failure or arrhythmia, even if the patient is not experiencing any problems.

Conflict of Interest

None declared.

\section{Acknowledgment}

The authors are very grateful to Dr. John Thomson from Leeds for his help to prepare this manuscript.

\section{References}

1 Rychik J, Atz AM, Celermajer DS, et al; American Heart Association Council on Cardiovascular Disease in the Young and Council on Cardiovascular and Stroke Nursing. Evaluation and management of the child and adult with Fontan circulation: a scientific statement from the American Heart Association. Circulation 2019;140(06CIR0000000000000696

2 Gewillig M, van de Bruaene A. FUELing the search for medical therapies in late Fontan failure. Circulation 2020;141(08): 652-654

3 Fontan F, Baudet E. Surgical repair of tricuspid atresia. Thorax 1971;26(03):240-248

4 Veldtman GR, Opotowsky AR, Wittekind SG, et al. Cardiovascular adaptation to the Fontan circulation. Congenit Heart Dis 2017;12 (06):699-710

5 Gewillig M, Goldberg DJ. Failure of the Fontan circulation. Heart Fail Clin 2014;10(01):105-116

6 Khairy P, Van Hare GF, Balaji S, et al. PACES/HRS expert consensus statement on the recognition and management of arrhythmias in adult congenital heart disease: developed in partnership between the Pediatric and Congenital Electrophysiology Society (PACES) and the Heart Rhythm Society (HRS). Heart Rhythm 2014;11(10): e102-e165 
7 Huntley GD, Deshmukh AJ, Warnes CA, Kapa S, Egbe AC. Longitudinal outcomes of epicardial and endocardial pacemaker leads in the adult Fontan patient. Pediatr Cardiol 2018;39(07):1476-1483

8 Helguera ME, Maloney JD, Woscoboinik JR, et al. Long-term performance of epimyocardial pacing leads in adults: comparison with endocardial leads. Pacing Clin Electrophysiol 1993;16(3 Pt 1):412-417

9 Zartner P, Handke R, Photiadis J, Brecher AM, Schneider MB. Performance of an autonomous telemonitoring system in children and young adults with congenital heart diseases. Pacing Clin Electrophysiol 2008;31(10):1291-1299

10 Nagel B, Janousek J, Koestenberger M, et al. Remote monitoring leads to early recognition and treatment of critical arrhythmias in adults after atrial switch operation for transposition of the great arteries. Circ J 2014;78(02):450-456

11 Sharma V, Burkhart HM, Cetta F, Hagler DJ, Phillips SD, Dearani JA. Fontan conversion to one and one half ventricle repair. Ann Thorac Surg 2012;94(04):1269-1274, discussion 1274

12 Janoušek J, Kovanda J, Ložek M, et al. Cardiac resynchronization therapy for treatment of chronic subpulmonary right ventricular dysfunction in congenital heart disease. Circ Arrhythm Electrophysiol 2019;12(05):e007157

13 Atz AM, Zak V, Mahony L, et al; Pediatric Heart Network Investigators. Longitudinal outcomes of patients with single ventricle after the Fontan procedure. J Am Coll Cardiol 2017;69(22): 2735-2744

14 Williams RV, Travison T, Kaltman JR, et al; Pediatric Heart Network Investigators. Comparison of Fontan survivors with and without pacemakers: a report from the Pediatric Heart Network Fontan Cross-Sectional Study. Congenit Heart Dis 2013;8(01):32-39

15 Gewillig M, Brown SC, van de Bruaene A, Rychik J. Providing a framework of principles for conceptualising the Fontan circulation. Acta Paediatr 2020;109(04):651-658

16 Koole MAC, Kauw D, Winter MM, et al. First real-world experience with mobile health telemonitoring in adult patients with congenital heart disease. Neth Heart J 2019;27(01):30-37

17 Balaji S, Daga A, Bradley DJ, et al. An international multicenter study comparing arrhythmia prevalence between the intracardiac lateral tunnel and the extracardiac conduit type of Fontan operations. J Thorac Cardiovasc Surg 2014;148(02):576-581

18 Tsao S, Deal BJ, Backer CL, Ward K, Franklin WH, Mavroudis C. Device management of arrhythmias after Fontan conversion. J Thorac Cardiovasc Surg 2009;138(04):937-940

19 Zartner PA, Mini N, Vergnat M, Momcilovic D, Schneider MB, Dittrich S. Performance of epimyocardial leads in patients with a single ventricle circulation. Pacing Clin Electrophysiol 2021;44 (05):903-910 